Manejo y aprovechamiento de recursos

\title{
Comparación morfológica entre poblaciones silvestres y manejadas de Opuntia atropes (Cactaceae) en Michoacán, México
}

\section{Morphological comparison between wild and cultivated populations of Opuntia atropes (Cactaceae) in Michoacán, Mexico}

\author{
Diana M. López-Gutiérrez ${ }^{\mathrm{a}}$, Juan A. Reyes-Agüero ${ }^{\mathrm{b}}$, Alejandro Muñoz ${ }^{\mathrm{c}}$, \\ Javier Robles ${ }^{a}$ y Eduardo Cuevas ${ }^{\mathrm{a}, *}$ \\ ${ }^{a}$ Facultad de Biología, Universidad Michoacana de San Nicolás de Hidalgo, Av. Francisco J. Mújica s/n, Ciudad Universitaria, 58000 Morelia, Michoacán, México \\ ${ }^{\mathrm{b}}$ Instituto de Investigación en Zonas Desérticas, Universidad Autónoma de San Luis Potosí, Altair 200, Fraccionamiento del Llano, 78377 San Luis Potosí, \\ San Luis Potosí, México \\ ${ }^{\mathrm{c}}$ Departamento de Ecología, Universidad de Guadalajara, Km 15.5 carretera Guadalajara Nogales, 44100 Zapopan, Jalisco, México
}

Recibido el 20 de febrero de 2015; aceptado el 27 de agosto de 2015

Disponible en Internet el 10 de noviembre de 2015

\begin{abstract}
Resumen
En México hay una antigua tradición de consumo de tallos y frutos de Opuntia (nopales), ya sean silvestres o en proceso de domesticación, que son importantes en la dieta de habitantes del centro de la República. Aun cuando Opuntia ficus-indica es la especie más cultivada en el país, hay otras especies de importancia regional, que han sido menos estudiadas como $O$. atropes, nativa del centro-occidente de México, muy apreciada por sus cladodios inmaduros o nopalitos. Se evaluó la variabilidad morfológica de plantas silvestres, toleradas, de huerto y de plantación de O. atropes, para establecer si esta especie se encuentra en proceso de domesticación, además de determinar las características que se han modificado. Se seleccionaron 3 zonas de muestreo en Michoacán, con una muestra total de 120 individuos. Después de analizar 14 variables morfológicas, se registró la divergencia morfológica entre plantas con y sin manejo. Los resultados muestran que los nopales cultivados presentaron cladodios inmaduros y maduros de mayor dimensión, con más areolas e hileras de areolas, y menos espinas y glóquidas. A partir de las diferencias reportadas y la amplia propagación de su cultivo en la región, se señala que $O$. atropes se encuentra en un proceso evolutivo de domesticación.

Derechos Reservados @ 2015 Universidad Nacional Autónoma de México, Instituto de Biología. Este es un artículo de acceso abierto distribuido bajo los términos de la Licencia Creative Commons CC BY-NC-ND 4.0.
\end{abstract}

Palabras clave: Domesticación; Manejo de plantas; Nopales; Variación morfológica

\section{Abstract}

Mexico has an ancient tradition of consumption of stems and fruits of Opuntia, of wild plants or plants in different degree of domestication. Even though Opuntia ficus-indica is the most cultivated species, there are other Opuntia species locally consumed and less well studied. This is the case of $O$. atropes native of Mexico and appreciated for its edible young cladodes "nopalitos". This study evaluated the morphological variability of wild, tolerated, of home gardens and commercial plantations, in order to know whether $O$. atropes is in the process of domestication and to explore which characteristics have been modified. Three study sites were selected in the state of Michoacán where a total of 120 plants were sampled. Fourteen morphological variables were analyzed using univariate and multivariate methods. A morphological divergence between individuals from managed and non-managed populations was observed. Inmature and mature cladodes from cultivated cactus pears were bigger, with more areoles

\footnotetext{
* Autor para correspondencia.

Correo electrónico: eduardocuevas5@gmail.com (E. Cuevas).

La revisión por pares es responsabilidad de la Universidad Nacional Autónoma de México.
} 
and with less spines and glochids. These differences as well as its wide cultivated extension in Michoacán suggests that $O$. atropes is going through an evolutionary process of domestication.

All Rights Reserved @ 2015 Universidad Nacional Autónoma de México, Instituto de Biología. This is an open access item distributed under the Creative Commons CC License BY-NC-ND 4.0.

Keywords: Domestication; Plant management; Cactus pear; Morphological variation

\section{Introducción}

En México, como en otros países de Mesoamérica, hay una larga tradición en el aprovechamiento de especies de plantas silvestres y/o en proceso de domesticación, particularmente en zonas rurales (Ashworth, Quesada, Casas, Aguilar y Oyama, 2009; Bravo-Hollis, 1978). Durante este tiempo, el hombre ha seleccionado ciertas características de acuerdo con sus propios intereses y necesidades, lo cual ha derivado en variedades manejadas, tanto de plantas como de animales, en las que se expresan algunas características distintas a las de sus parientes silvestres, siendo las morfológicas las más evidentes (Casas, Caballero, Valiente-Banuet, Soriano y Dávila, 1999).

En la familia Cactaceae, el género Opuntia agrupa entre 150 y 180 especies (Majure et al., 2012) e incluye especies útiles por sus frutos y tallos comestibles. Se estima que desde hace 14,000 años se consumen plantas de este género en el país (Casas y Barbera, 2002). Además, los nopales pueden encontrarse de forma silvestre, en huertos o solares de traspatio $\mathrm{y}$ en plantaciones comerciales (Figueroa, Aguirre y García, 1980; Reyes-Agüero, Aguirre y Flores, 2005). Estos tipos de ambientes se pueden relacionar con distintas intensidades de manejo y con cambios morfológicos debido al proceso de domesticación (Colunga, Hernández y Castillo, 1986).

Opuntia ficus-indica es la especie de nopal más cultivada en México y en el mundo, y en ella se pueden distinguir morfológicamente variedades que se utilizan para obtener frutos y nopalitos para su consumo (Reyes-Agüero, Aguirre y Carlín, 2004). Sin embargo, hay otras especies menos estudiadas que suelen ser consumidas y que se propagan por la calidad de sus nopalitos. Tal es el caso de $O$. atropes, la cual, de acuerdo con Colunga et al. (1986), parece estar diferenciada morfológicamente de las especies afines en los caracteres de los cladodios, los cuales durante el proceso de domesticación son preferidos por el hombre.

O. atropes es una especie endémica de México (Bravo-Hollis, 1978), conocida como «nopal blanco» (Colunga et al., 1986; Reyes-Agüero, Aguirre, Castelán y González, 2009), y se le ha ubicado en un proceso incipiente de domesticación, por tener una morfología intermedia entre los nopales silvestres y los más modificados por el hombre (Reyes-Agüero et al., 2005). Por lo tanto, si ha ocurrido selección artificial en $O$. atropes (López Gutiérrez, 2014), se esperarían diferencias morfológicas entre los individuos de poblaciones silvestres, toleradas, de huerto y plantación, condiciones de manejo de esta especie en la zona de estudio. El objetivo central de este estudio fue evaluar la variabilidad morfológica de $O$. atropes en dichas condiciones en el centro-norte de Michoacán.

\section{Materiales y métodos}

$O$. atropes es conocida como nopal blanco y en la parte norte de Michoacán presenta las siguientes características: cactácea de arbustiva a arborescente, de 1 a $4 \mathrm{~m}$ de altura, con tronco negro, escamoso y con espinas formando rosetas. Las plantas tienen cladodios pubescentes, de obovados a oblongos, de 15 a $30 \mathrm{~cm}$ de longitud, de un verde no muy oscuro, más claros cuando jóvenes. Espinas blancas de 1 a 4 por areola, con la base y la punta amarillenta, de $1-3 \mathrm{~cm}$, prevalentemente difusas. Presenta botones de color rosa intenso y flores naranja que se tornan amarillas. Sus frutos son obovados, verde blanquecino y pequeños, y las areolas de los cladodios y del pericarpelo de las flores y frutos poseen abundantes glóquidas amarillas (López-Gutiérrez, 2014).

A partir del estudio etnobotánico de López-Gutiérrez (2014) se ubicaron 3 zonas en los alrededores de Morelia (tabla 1). En dichas zonas se identificaron poblaciones de $O$. atropes en las siguientes 4 condiciones: silvestres, toleradas - en predios con cambio de uso de suelo-, en huertos caseros y en plantaciones comerciales. Posteriormente, el Dr. Salvador Arias-Montes, del Instituto de Biología de la Universidad Nacional Autónoma de México, confirmó la correcta identidad de las muestras de O. atropes. Entre abril y junio del 2013, cuando las plantas presentaban producción de nuevos cladodios «nopalitos», se seleccionaron 10 plantas por zona y por condición, para un total de 120 individuos. De cada planta se recolectaron un nopalito y un cladodio maduro, en adelante llamado solo «cladodio».

Los cladodios se midieron en fresco, con ayuda de un vernier digital y una cinta flexible. Las estructuras pequeñas se observaron con ayuda de una lupa $10 \times$. En cada nopalito y cladodio se midieron 12 variables cuantitativas y 2 cualitativas que pudieran haber sufrido cambios por selección artificial (tabla 2). Debido a que los frutos de $O$. atropes no son apreciados por los campesinos por ser pequeños y con abundantes glóquidas, no se registraron sus características morfológicas.

Se realizaron análisis de componentes principales (ACP) utilizando las 14 variables de respuesta y analizando por separado los nopalitos y los cladodios. Posteriormente, con la finalidad de saber si las características morfológicas difieren respecto a las 4 condiciones de manejo, silvestres, toleradas, de huerto y plantaciones, y al sitio, se realizó un análisis de varianza multivariado (Manova). Para ello, se utilizaron los valores de los «factor scores» de los 2 primeros componentes del ACP realizado a los cladodios y nopalitos, respectivamente, que incluía la combinación lineal de las variables de respuesta que integran estos «factor score», incluyendo en el análisis el sitio y la condición de manejo como factores fijos, así como su respectiva interacción. 
Tabla 1

Ubicación de las 3 zonas de muestreo.

\begin{tabular}{|c|c|c|c|c|}
\hline Municipio & Localidad & Latitud (N) & Longitud $(\mathrm{O})$ & Altitud (m) \\
\hline \multirow[t]{3}{*}{ Morelia } & La Concepción & $19^{\circ} 42^{\prime} 29^{\prime \prime}$ & $101^{\circ} 19^{\prime} 11^{\prime \prime}$ & 2,150 \\
\hline & Joya de Buenavista & $19^{\circ} 37^{\prime} 35^{\prime \prime}$ & $101^{\circ} 18^{\prime} 44^{\prime \prime}$ & 2,150 \\
\hline & Joya de la Huerta & $19^{\circ} 36^{\prime} 52^{\prime \prime}$ & $101^{\circ} 18^{\prime} 57^{\prime \prime}$ & 2,150 \\
\hline \multirow[t]{2}{*}{ Tarímbaro } & Cañada de los Sauces & $19^{\circ} 49^{\prime} 55^{\prime \prime}$ & $101^{\circ} 13^{\prime} 09^{\prime \prime}$ & 2,040 \\
\hline & El Curiro & $19^{\circ} 50^{\prime} 24^{\prime \prime}$ & $101^{\circ} 12^{\prime} 24^{\prime \prime}$ & 2,060 \\
\hline
\end{tabular}

Las localidades Joya de Buenavista y Joya de la Huerta se consideraron pertenecientes a la misma zona de muestreo por su cercanía geográfica, al igual que las localidades Cañada de los Sauces y El Curiro.

Además, se realizaron pruebas de contraste múltiples en los factores que aportaron diferencias. Finalmente, se realizaron Anova y pruebas múltiples de medias (Tukey) en algunas de las variables estimadas que más se relacionaban con el proceso de domesticación, como el tamaño de los cladodios y el número de espinas. Los datos fueron analizados con el programa estadístico JMP 7 (versión 7; SAS Institute, Inc., Cary, NC, USA).

\section{Resultados}

El ACP de los nopalitos y de los cladodios ordenó dimensionalmente a los individuos cultivados - de huerto y plantación, en adelante denominados «manejados»- de los no cultivados —silvestres y tolerados, llamados «no manejados» (fig. 1). Además, en el ACP realizado con los nopalitos únicamente se observan 2 individuos que se traslapan (fig. 1). Esta separación se relaciona con el primer componente principal tanto en los nopalitos como en los cladodios. En dicho sentido, el componente principal 1 de los nopalitos explica el $36 \%$ de la variación y el $33 \%$ el del cladodio maduro. A pesar de que

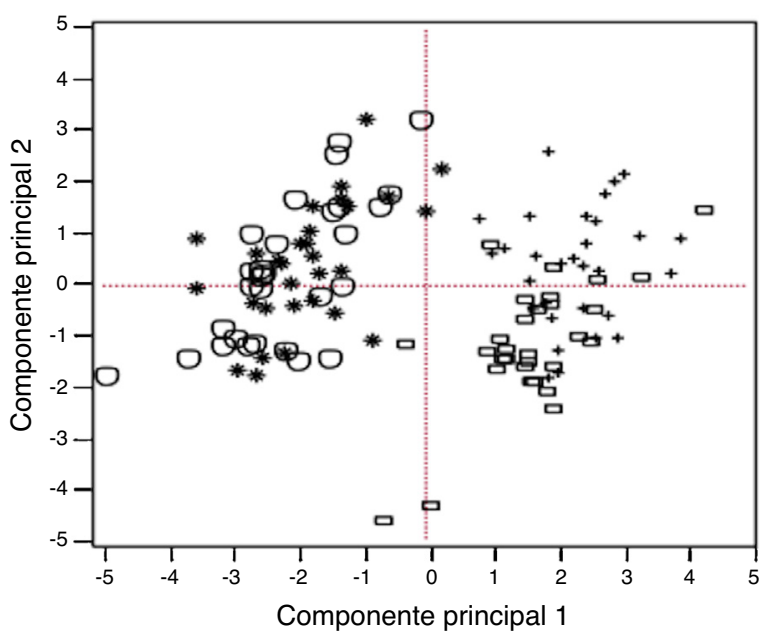

Figura 1. Análisis de componentes principales utilizando los nopalitos. Se muestra el análisis interpretado por la condición de manejo de las plantas. Los individuos de poblaciones no manejadas - silvestres (representados por asteriscos) y tolerados (representados por óvalos) - se localizan principalmente del lado izquierdo a lo largo del componente principal 1 (eje X) y los individuos con manejo - huerto (representados por rectángulos) y plantaciones(por cruces)se encuentran principalmente a la derecha del mismo eje. Al hacer el análisis por localidad de origen de las plantas, no se observaron patrones de agrupación debidas al sitio. son porcentajes relativamente bajos, este componente permite separar visualmente a los individuos cultivados de los no cultivados (fig. 1). El modelo general del Manova utilizando los cladodios aportó contraste $\left(\mathrm{F}_{4}, 114=4.73, p<0.0001\right)$ y hubo diferencias entre las condiciones de manejo $\left(\mathrm{F}_{3},{ }_{114}=4.69, p<\right.$ $0.0001)$, pero no entre localidades $\left(\mathrm{F}_{2}, 114=0.03, p=0.11\right)$. De manera similar, en el caso de los nopalitos el modelo general del Manova también indicó diferencias $\left(\mathrm{F}_{5}, 114=0.17, p=0.002\right)$ y se obtuvo contraste entre las condiciones de manejo $\left(\mathrm{F}_{3}, 114\right.$ $=4.69, p=0.007)$, pero no entre localidades $\left(\mathrm{F}_{2,114}=0.006\right.$, $p=0.06)$. Finalmente, en las pruebas de contraste realizadas tanto con los nopalitos como en los cladodios entre las distintas condiciones, en ambos casos se registraron diferencias entre las plantas silvestres y las manejadas, es decir, de huerto y de plantación. Las plantas toleradas presentaron afinidad con las plantas de huerto, pero no con las de plantaciones comerciales (tabla 3). Los nopalitos manejados presentan un margen más entero -asociado a podarios menos prominentes-, son más largos y anchos, con más hileras de areolas, mayor cantidad de areolas en el margen y en la cara, menos areolas con espinas, menos espinas por areola, menos espinas y glóquidas menos abundantes (tabla 2). Los nopalitos manejados son proporcionalmente más delgados que los no manejados (tabla 2). Al comparar los cladodios maduros manejados con los no manejados, se encontraron casi las mismas diferencias que en los nopalitos (tabla 2).

\section{Discusión}

A pesar de la amplia relevancia que tiene el género Opuntia en México, pocos trabajos han abordado los procesos de domesticación en este género (Colunga et al., 1986; Reyes-Agüero et al., 2004, 2005). A diferencia de trabajos previos que han comparado los cambios morfológicos entre especies (Colunga et al., 1986; Reyes-Agüero et al., 2005) o entre distintas variedades cultivadas de una misma especie (Reyes-Agüero et al., 2004), este trabajo comparó la morfología de individuos manejados y no manejados de una misma especie de Opuntia. Además, observamos que en el área de estudio $O$. atropes es muy apreciada como nopalito y ampliamente cultivada.

De manera general, se detectaron diferencias morfológicas entre los individuos silvestres respecto de los de plantaciones y de huerto, tanto en nopalitos como en cladodios (tabla 3). Además, hay contraste entre los nopalitos de huertos y los de plantación (tabla 3), aunque dichas diferencias no se observaron en los cladodios. Esta diferencia entre los nopalitos puede deberse a que la intensidad de manejo actual es distinta, 
Tabla 2

Valores promedio \pm un error estándar de las 14 variables medidas en los nopalitos y en los cladodios bajo las 4 condiciones: no manejados —silvestre y tolerado—y manejados —huerto y plantación.

\begin{tabular}{|c|c|c|c|c|c|c|c|c|}
\hline & \multicolumn{4}{|c|}{ Nopalitos } & \multicolumn{4}{|c|}{ Cladodios } \\
\hline & Silvestre & Tolerado & Huerto & Plantación & Silvestre & Tolerado & Huerto & Plantación \\
\hline Largo del cladodio & $\mathbf{1 3 . 0 7} \pm \mathbf{0 . 3 b}$ & $14.05 \pm 0.5 b$ & $18.52 \pm 0.84 \mathrm{a}$ & $18.97 \pm \mathbf{0 . 7 a}$ & $22.01 \pm 0.5 c$ & $21.6 \pm 0.8 \mathrm{c}$ & $28.2 \pm 0.9 b$ & $31.5 \pm 0.7 a$ \\
\hline Ancho del cladodio & $7.22 \pm \mathbf{0 . 1 6 b}$ & $7.8 \pm 0.23 \mathrm{ab}$ & $8.2 \pm 0.28 \mathrm{a}$ & $8.3 \pm 0.21 \mathrm{a}$ & $12.2 \pm 0.32 b$ & $12 \pm 0.31 b$ & $12.7 \pm 0.35 \mathrm{ab}$ & $13.54 \pm 0.27 a$ \\
\hline Grosor del cladodio & $0.45 \pm 0.01$ & $0.52 \pm 0.02$ & $0.49 \pm 0.02$ & $0.5 \pm 0.02$ & $1.04 \pm 0.06$ & $1.1 \pm 0.06$ & $1.09 \pm 0.05$ & $1.15 \pm 0.04$ \\
\hline Distancia entre areolas & $2.36 \pm 0.07$ & $2.71 \pm 0.1$ & $2.63 \pm 0.12$ & $2.57 \pm 0.07$ & $3.9 \pm 0.1$ & $3.8 \pm 0.15$ & $3.9 \pm 0.13$ & $4.3 \pm 0.14$ \\
\hline Número de hileras de areolas & $6 \pm 0.18$ & $5.63 \pm 0.18$ & $7.1 \pm 0.21$ & $7.7 \pm 0.26$ & $5.8 \pm 0.17$ & $5.9 \pm 0.1$ & $6.6 \pm 0.1$ & $7.2 \pm 0.16$ \\
\hline Número de areolas en el margen & $33.23 \pm 0.9$ & $33.46 \pm 0.92$ & $40.56 \pm 1.06$ & $41.2 \pm 0.93$ & $29.6 \pm 0.77$ & $28.7 \pm 1.07$ & $33.6 \pm 0.93$ & $36.9 \pm 0.9$ \\
\hline Número de areolas en una cara & $23.3 \pm 1 b$ & $22.16 \pm 0.91 b$ & $31.46 \pm 1.21 \mathrm{a}$ & $35.5 \pm 1.4 a$ & $23.36 \pm 0.78 c$ & $24.13 \pm 1.37 \mathrm{c}$ & $29.06 \pm 0.8 b$ & $33.23 \pm 0.86 a$ \\
\hline Densidad de areolas en $25 \mathrm{~cm}^{2}$ & $6.4 \pm 0.2$ & $5.9 \pm 0.4$ & $6.26 \pm 0.5$ & $5.6 \pm 0.2$ & $2.7 \pm 0.12$ & $2.86 \pm 0.1$ & $2.93 \pm 0.14$ & $2.36 \pm 0.08$ \\
\hline Número de areolas con espinas & $13.8 \pm 0.59 a$ & $13.8 \pm 1.13 \mathrm{a}$ & $0.7 \pm 0.3 b$ & $1.4 \pm 0.59 b$ & $19.2 \pm 0.7 \mathrm{a}$ & $19.3 \pm 1.54 \mathrm{a}$ & $3 \pm 0.6 b$ & $6.6 \pm 1 \mathbf{b}$ \\
\hline Número de espinas por areola & $1.16 \pm 0.06$ & $1.7 \pm 0.2$ & $0.66 \pm 0.04$ & $0.16 \pm 0.09$ & $2.56 \pm 0.13$ & $2.93 \pm 0.19$ & $0.1 \pm 0.05$ & $0.8 \pm 0.14$ \\
\hline Número de espinas menores a $1 \mathrm{~cm}$ & $0.5 \pm 0.1$ & $0.9 \pm 0.21$ & $0.66 \pm 0.04$ & $0.13 \pm 0.09$ & $0.66 \pm 0.14$ & $0.76 \pm 0.18$ & $0.6 \pm 0.04$ & $0.36 \pm 0.12$ \\
\hline Número de espinas de 1 a $3 \mathrm{~cm}$ & $0.73 \pm 0.1$ & $0.76 \pm 0.13$ & 0 & o & $1.83 \pm \mathbf{0 . 1 5}$ & $1.93 \pm 0.11$ & $0.03 \pm 0$ & $0.46 \pm 0.09$ \\
\hline Tipo de margen ${ }^{\mathrm{a}}$ & $2.7 \pm 0.07$ & $2.5 \pm 0.12$ & $2 \pm 0.06$ & $2.13 \pm 0.07$ & $1.96 \pm 0.08$ & $2 \pm 0.11$ & $1.53 \pm 0.09$ & $1.46 \pm 0.09$ \\
\hline Abundancia de glóquidas ${ }^{\mathrm{b}}$ & $1.83 \pm 0.14$ & $1.73 \pm 0.20$ & $1.03 \pm 0.03$ & $1.10 \pm 0.07$ & $2.13 \pm 0.16$ & $217 \pm 0.14$ & $1.33 \pm 0.15$ & $\mathbf{0 . 7} \pm \mathbf{0 . 0 8}$ \\
\hline
\end{tabular}

Los valores en negritas corresponden a las características donde las diferencias entre la condición silvestre y plantación fueron más evidentes, como el gigantismo -largo y ancho del cladodio- y la pérdida de

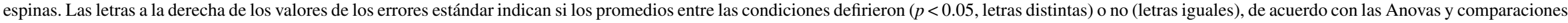
múltiples de medias realizadas. Variables cualitativas.

a Tipo de margen: 1 , entero; 2 , ondulado y 3 , sinuado.

b Abundancia de glóquidas: 1 , sin glóquidas; 2 , pocas glóquidas; 3 , glóquidas medianamente abundantes y 4, glóquidas abundantes. 
Tabla 3

Valores de probabilidad de las pruebas de contraste realizadas entre las distintas condiciones de manejo para los nopalitos (A) y cladodios (B).

\begin{tabular}{|c|c|c|c|}
\hline \multicolumn{4}{|c|}{ A } \\
\hline & Plantación & Tolerada & Huerto \\
\hline & & & Plantación \\
\hline Tolerada & 0.32 & & \\
\hline Huerto & 0.01 & 0.11 & \\
\hline Silvestre & 0.03 & 0.07 & 0.001 \\
\hline \multicolumn{4}{|c|}{ B } \\
\hline & Plantación & Tolerada & Huerto \\
\hline & & & Plantación \\
\hline Tolerada & 0.0001 & & \\
\hline Huerto & 0.21 & 0.11 & \\
\hline Silvestre & 0.0001 & 0.07 & 0.0001 \\
\hline
\end{tabular}

Las comparaciones se realizaron a partir del modelo de Manova, en el que se utilizaron como variables de respuesta los «factor values» de los 2 primeros componentes del ACP en el que se utilizaron las 14 variables originales. No se muestran las comparaciones entre localidades, ya que no hubo diferencias.

también a que los individuos de las plantaciones son de cultivares de reciente introducción, provenientes de plantas con características distintas (López-Gutiérrez, 2014). Estos cambios pueden indicar también que las plantas presentan un grado distinto de domesticación. Las diferencias morfológicas detectadas entre las poblaciones silvestres y toleradas de los cladodios (tabla 3B) pueden ser debidas al manejo incipiente de estas últimas, ya que cuando se realiza un cambio de uso de suelo se dejan en pie algunas plantas de nopal blanco. Es posible que los individuos que permanecen en pie se debe a sus características individuales, lo cual podría explicar por qué son morfológicamente distintos de los silvestres, aunque los moradores refieren que se dejan en pie las plantas en los lindes de sus predios (López-Gutiérrez, 2014). Se observó una afinidad en los nopalitos y los cladodios tolerados con los de huerto, pero solo los nopalitos presentan similitudes entre los tolerados y los de plantación. Las diferencias entre los cladodios de plantación y tolerados podría indicar que las plantas toleradas se encuentran en un estado incipiente de domesticación que no se observa siempre en los nopalitos (tabla 3 ).

De manera similar a lo documentado previamente para especies en cultivo (Colunga et al., 1986; Reyes-Agüero et al., 2004, 2005), O. atropes presenta gigantismo, pues el tamaño de los cladodios manejados fue mayor que en los no manejados. El gigantismo en las plantaciones, aumento en el largo del cladodio, se detectó en la misma magnitud (69\%) tanto en nopalitos como en cladodios, respecto a las plantas silvestres (tabla 2). En los nopalitos, a pesar del incremento en el largo como en el ancho del cladodio, el grosor no varió. Por ello, los nopalitos son proporcionalmente menos gruesos, lo que concuerda con lo señalado por los entrevistados en la región (López-Gutiérrez, 2014), quienes comentaron que los nopalitos de huerto son más delgados.

Otra característica evidente fue la reducción y/o pérdida de espinas en los nopales cultivados, fenómeno que al igual que el gigantismo es registrado como resultado de la domesticación de muchas plantas (Baker, 1972; Hawkes, 1983; Reyes-Agüero et al., 2005). El haber utilizado cladodios inmaduros y maduros permitió distinguir cómo la pérdida de estructuras de defensa, número de areolas con espinas, fue más evidente en los nopalitos de las plantaciones que en los nopales maduros de la misma condición al contrastarlos con los de plantas silvestres. Esto puede ser el resultado de una mayor presión de selección en las características de los nopalitos, que es la etapa en la que son consumidos como verdura. En este sentido, Colunga et al. (1986) señalan que una de las características apreciadas en $O$. atropes es la prominencia de los podarios en los nopalitos, lo que facilita su pelado. Sin embargo, en las poblaciones aquí analizadas los podarios están disminuidos en el morfotipo cultivado en comparación con el silvestre. Por otro lado, Reyes-Agüero et al. (2005) registraron que en los nopales con mayor grado de domesticación tanto el número de hileras como la densidad de areolas son más bajos. Sin embargo, en $O$. atropes se encontró un fenómeno distinto, pues el número de hileras aumentó en las plantas manejadas, permaneciendo constante la distancia entre las areolas, por lo cual estas se presentan en mayor número en comparación con las plantas no manejadas.

En el caso de las variedades usadas para nopalito de Opuntia ficus-indica, las características importantes son: cladodios pequeños — con relación a los nopales tuneros- y con varias areolas con espinas, nopalitos con algunas espinas y espinas setosas en sus areolas (Reyes-Agüero et al., 2005). En O. atropes se han seleccionado cladodios más grandes, con areolas con pocas espinas o sin ellas, particularmente en los nopalitos.

Se requieren estudios posteriores para conocer si todos los cambios morfológicos detectados presentan una base genética o se deben a la plasticidad fenotípica ocasionada por las diferentes condiciones de cultivo, así como un estudio más amplio a lo largo de su distribución para conocer el posible manejo de esta especie. La serie de características que se han modificado en los nopales cultivados, así como la amplia extensión de sus cultivos, indican que $O$. atropes se encuentra en un proceso evolutivo de domesticación en la zona de estudio.

\section{Agradecimientos}

A la Coordinación de la Investigación Científica de la Universidad Michoacana de San Nicolás de Hidalgo por el financiamiento otorgado para la realización de este estudio. D. M. López-Gutiérrez agradece al Consejo Nacional de Ciencia y Tecnología la beca otorgada durante su maestría. A los 2 revisores anónimos, así como a la editora asociada por sus valiosos comentarios, los cuales mejoraron sustancialmente el trabajo.

\section{Referencias}

Ashworth, L., Quesada, M., Casas, A., Aguilar, R. y Oyama, K. (2009). Pollinator-dependent food production in Mexico. Biological Conservation, 142, 1050-1057.

Baker, H. G. (1972). Human influence on plant evolution. Economic Botany, 26, $32-43$.

Bravo-Hollis, H. (1978). Las cactáceas de México. Tomo I. México. D.F.: Universidad Nacional Autónoma de México.

Casas, A., Caballero, J., Valiente-Banuet, A., Soriano, J. A. y Dávila, P. (1999). Morphological variation and the process of domestication of Stenocereus 
stellatus (Cactaceae) in central Mexico. American Journal of Botany, 86 , 522-533.

Casas, A. y Barbera, G. (2002). Mesoamerican domestication and diffusion. En P. S. Nobel (Ed.), Cacti: biology and uses (pp. 143-162). Berkeley: University of California Press.

Colunga, P., Hernández, E. y Castillo, A. (1986). Variación morfológica, manejo agrícola tradicional y grado de domesticación de Opuntia spp. en el Bajío guanajuatense. Agrociencia, 65, 7-44.

Figueroa, H. F., Aguirre, J. R. y García, E. (1980). Estudio de las nopaleras cultivadas y silvestres sujetas a recolección para el mercado en el altiplano potosino-zacatecano. México, D.F: Avances en la Enseñanza e Investigación. Colegio de Posgraduados.

Hawkes, J. G. (1983). The diversity of crop plants. Cambridge: Harvard University Press.

López-Gutiérrez, D. M. (2014). Variación morfológica de poblaciones silvestres y manejadas de Opuntia atropes en la cuenca de Cuitzeo. Tesis de maestría. Facultad de Biología, Universidad Michoacana de San Nicolás de Hidalgo.
Majure, L. C., Puente, R., Griffith, M. P., Judd, W. S., Soltis, P. S. y Soltis, D. E. (2012). Phylogeny of Opuntia S.S (Cactaceae): Clade delimitation, geographic origins, and reticulate evolution. American Journal of Botany, 99, 847-864

Reyes-Agüero, J. A., Aguirre, R. J. R. y Carlín, C. F. (2004). Análisis preliminar de la variación morfológica de 38 variantes mexicanas de Opuntia ficusindica (L.) Miller. En F. G. Esparza, Z. R. D. Valdez, y S. J. G. Méndez (Eds.), El nopal, tópicos de actualidad (pp. 21-47). México, D.F.: Universidad Autónoma de Chapingo y Colegio de Posgraduados.

Reyes-Agüero, J. A., Aguirre, J. R. y Flores, J. L. (2005). Variación morfológica de Opuntia (Cactaceae) en relación con su domesticación en la altiplanicie meridional de México. Interciencia, 30, 476-484.

Reyes-Agüero, J. A., Aguirre, J. R., Castelán, F. y González, A. (2009). Catálogo de las principales variantes silvestres y cultivadas de Opuntia en la altiplanicie meridional de México. México, D.F: Secretaría de Agricultura, Ganadería, Desarrollo Rural, Pesca y Alimentación y Comisión Nacional de Ciencia y Tecnología. 